\title{
A Note on the Origin of the Twin Prime Conjecture
}

\section{by William Dunham}

\author{
Department of Mathematics \& Computer Science, Muhlenberg College; \\ Department of Mathematics, Harvard University (visiting 2012-2013)
}

More than any other branch of mathematics, number theory features a collection of famous problems that took centuries to be proved or that remain unresolved to the present day.

Among the former is Fermat's Last Theorem. This states that it is impossible to find a whole number $n \geq 3$ and whole numbers $x, y$, and $z$ for which $x^{n}+y^{n}=z^{n}$. In 1640, as all mathematicians know, Fermat wrote this in a marginal note and claimed to have "an admirable proof" that, alas, did not fit within the space available. The result remained unproved for 350 years until Andrew Wiles, with assistance from Richard Taylor, showed that Fermat was indeed correct.

On the other hand, there is the Goldbach conjecture, which asserts that every even number greater than 2 can be expressed as the sum of two primes. Goldbach shared this with Euler in 1742. Euler responded that he believed the result was true, but "I cannot prove it." Today - two and a half centuries later - no one else has done so.

Alongside these two examples stands the twin prime conjecture (TPC). However, whereas the histories of Fermat's Last Theorem and the Goldbach conjecture are well known, the origins of the twin prime conjecture are more uncertain. The conjecture itself is easy to state. We define twin primes as those that differ by 2 . Thus 3 and 5 are twins, as are 11 and 13 as well as 41 and 43 . The twin prime conjecture says that there are infinitely many such pairs. As with the Goldbach conjecture, no one has yet been able to supply a proof.

Some questions arise naturally: Where did the conjecture come from? Whose name should we attach to it? And when did it join the ranks of the famous problems of number theory?

To answer these, we first look to the Comptes rendus of the French Academy of Sciences, dated 15 October, 1849, and to a paper titled "New Research on Prime Numbers" by Alphonse de Polignac. He was a French mathematician who lived from 1817 to 1890 . There seems to be little information about him, except that he was an alumnus of the École Polytechnique and served as a sous-lieutenant in the French artillery.
As the title suggests, de Polignac's paper was about prime numbers, and on p. 400 he stated two "theorems." It should be noted that he was not using the word in its modern sense. Rather, de Polignac confirmed the truth of these results by checking a number of cases and thereby called them "theorems." Of course, we would call them, at best, "conjectures." The true nature of these statements is suggested by the fact that de Polignac introduced them under the heading "Induction and remarks" as seen below, in a facsimile of his original text.

$$
\text { SIV. - Inductions at remarques. }
$$

" Théorème. Tout nombre pair est égal à la différence de denx nombres premiers conséculifs d'une infinité de manières ( $7^{\circ}$ Théorème du $\S \mathbb{H}$ ).

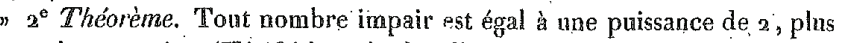
un nombre premier. (Vérifié jusqu'à 3 millions.)

De Polignac's first item says:

1st Theorem: Every even number is equal to the difference of two consecutive prime numbers in an infinitude of ways.

If we take his generic "even number" to be the specific number "2," then de Polignac's statement asserts the existence of an infinitude of twin primes. That is, the TPC becomes a special case of his conjecture.

Needless to say, de Polignac did not prove his "theorem" for 2 or for any other even number. As noted above, he simply convinced himself by checking examples. The question is whether we should regard this as the origin of the twin primes conjecture.

On the one hand, de Polignac's assertion certainly includes the TPC. But it is also true that he did not single out the twin prime case for special attention, nor did he assign to it a special name. For this reason, we might want to pause before assigning him credit.

His authority is, perhaps, further diminished by the second entry that appears in the excerpt:

2nd Theorem: Every odd number is equal to a power of two plus a prime number.

Further, de Polignac noted that his result had been "verified up to three million." 
This would be a lovely theorem indeed. It would decompose any odd number into the sum of a prime and a power of two, where the latter is surely the most "even" of all integers. As an example, we have the odd numbers $25=17+2^{3}$ and $41=37+2^{2}$

Unfortunately, his "theorem" is quite wrong. For one thing, it is hard to imagine how anyone in the days before computers could check such a statement for one-and-a-half million odd numbers. But, far worse, a counterexample is easy to find.

De Polignac admitted as much on pp. 738-739 of a later issue of the Comptes rendus of 1849. Perhaps to mitigate his own carelessness, he noted that Euler, in 1753 , had briefly entertained this same notion. But Euler quickly realized that the numbers 127 (an odd prime) and 959 (an odd composite) cannot be decomposed into the sum of a prime and a power of 2 .

Even as he acknowledged his error, de Polignac tried to excuse it. He observed that he had been "pressed to the extreme" by other duties. And he said that he had not been able "to do these calculations myself." Perhaps these excuses lessened the embarrassment of the moment.

In any case, it is clear that Alphonse de

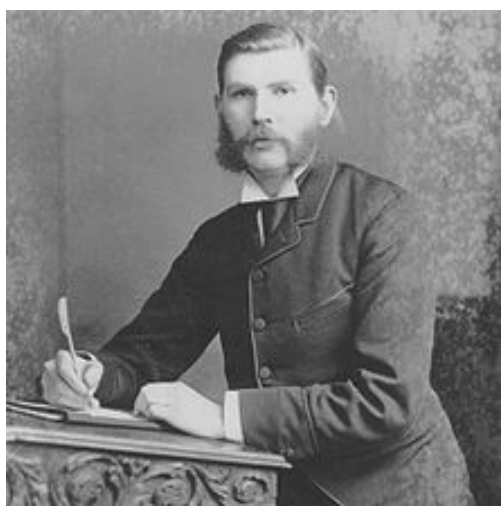

J.W.L. Glaisher Polignac was guilty of reaching conclusions much too hastily. But what, then, do we make of his conjecture about prime differences (i.e., his 1st Theorem)? Did it arise from similarly sloppy thinking? This possibility, combined with the fact that he never explicitly mentioned twin primes, suggests that de Polignac deserves at most partial credit for the TPC.

So we must jump ahead half a century to the British mathematician James Whitbread Lee Glaisher (18481928). Unlike de Polignac, about whom little is known, Glaisher has an extensive biography. He was a son of Trinity College, Cambridge, which he attended as an undergraduate and where he spent his entire professional career. Glaisher obviously had mathematical gifts, for he was Second Wrangler in 1871, was elected to the Royal Society four years later, and worked in both pure (number theory) and applied (astronomy) areas of mathematics.

Moreover, Glaisher was a proven leader, serving as president of the British Association for the Advancement of Science, the London Mathematical Society, and the Royal Astronomical Society. In 1908 he received the De Morgan Medal from the LMS, and in 1913 he won the Royal Society's Sylvester Medal.
He was also a tireless editor of mathematical journals. He edited the Quarterly Journal of Mathematics for decades and, remarkably, edited Messenger of Mathematics for 56 years!

But his accomplishments do not end there. Glaisher collected needlework, valentines, children's books, and, especially, pottery. This last collection grew so vast that he had to take extra rooms in Trinity College just to store his thousands of pots, vases, and other ceramic items. Upon his death, these were bequeathed to the Fitzwilliam Museum in Cambridge, where many pieces are now displayed as part of the Glaisher Collection.

Beyond this, he served as tutor to the young Ludwig Wittgenstein when the latter enrolled at Cambridge. He was a bicyclist who rose to become president of the local bicycle club. And he was an avid hiker who, according to reports, strode across the English countryside so fast that his breathless companions could not keep up.

Regarding mathematics, G. H. Hardy said of Glaisher, "...he was old-fashioned, but the best of his work is really good." As an example that is both old-fashioned and good, I cite a 1902 paper in which Glaisher sought the exact sum of the infinite series

$1+\frac{1}{2}-\frac{1}{3}+\frac{1}{4}-\frac{1}{5}-\frac{1}{6}+\frac{1}{8}+\frac{1}{9}-\frac{1}{10}+\frac{1}{11}-\frac{1}{12}-\frac{1}{13}+\frac{1}{15}+\cdots$

where, he explained, the sign of the fraction is " + " if the denominator is congruent to 1,2 , or $4(\bmod 7)$; the sign is " - " if the denominator is congruent to 3,5 , or $6(\bmod 7)$; and where no fraction appears if the denominator is a multiple of 7 . After manipulating formulas in a manner reminiscent of Euler, he established that this infinite series converges to $\frac{\pi}{\sqrt{7}}$, which is a most unlikely and remarkable answer.

For us, his paper of interest is "An Enumeration of Prime-Pairs," which was published in Messenger of Mathematics in 1879. In this article, Glaisher introduced a "prime-pair" as two primes "separated by only one number." Of course, these are what we call twin primes. He then counted all prime-pairs among the first million numbers, the second million numbers, etc., in order to get a sense of their distribution.

At that point, Glaisher made the critical observation, reproduced below from the original article:

32 Mr. GLAisher, AN ENUMERATION OF PRIME-PAIRS.

There can be little or no doubt that the number of primepairs is unlimited; but it would be interesting, though probably not easy, to prove this.

Here the infinitude of twin primes is mentioned explicitly. And here the mathematical community is challenged, at least implicitly, to prove it. As a consequence, we might regard Glaisher, not de Polignac, as the originator of the TPC. 
A few more names should be included in our story. In a 1916 article, the German mathematician Paul Stäckel used the words Primzahl-Zwillinge ("twin prime numbers"), which became more popular than "prime-pair." Three years later, in his landmark paper on the subject, Viggo Brun translated Stäckel's wording into French by calling these nombres premiers jumeaux ("twin primes"). By then, the terminology had become standard.

Brun's paper, by the way, was a classic of analytic number theory. As early as 1737 Euler had proved that the sum of the reciprocals of all primes is divergent. Brun, by contrast, showed that the infinite series formed by summing the reciprocals of the twin primes is convergent. Of course, this did not settle the question of their infinitude, for an infinite series can have a finite sum. But it stood alongside other landmarks of analytic number theory from the late 19th and early 20th centuries, including work by such great mathematicians as Jacques Hadamard, Charles-Jean Étienne Gustave Nicolas de la Vallée Poussin (who had too many names), G. H. Hardy and J. E. Littlewood (who had too few), and Srinivasa Ramanujan.

Late in the 20th century the TPC had another, and rather strange, claim to fame. In 1994, while using a computer to enumerate twin primes, the mathematician Thomas Nicely observed inconsistencies in his output. A little checking revealed the source: a flaw in the computer's Pentium processor. This flaw came to be known as the "Pentium FDIV bug." The processor's manufacturer, Intel, agreed to replace the chip for anyone who asked, and the resulting costs to the company were substantial. The story can be found in Barry Cipra's short piece "How Number Theory Got the Best of the Pentium Chip" in Science, Vol. 267 (1995), p. 175, but the critical point is that, had it not been for twin primes, this problem might never have been detected!

Recently, the mathematical world has been buzzing over a proof by Yitang Zhang that bears on the TPC. He showed that there is a finite constant $C$ with the property that infinitely many pairs of consecutive primes differ by $C$ or less. If $C=2$, we have the twin prime conjecture. His argument established the result for the considerably larger value of $C=70,000,000$, and it is for this reason that he called it a "weak" twin prime conjecture. Nonetheless, the work of Yitang Zhang is seen as a significant breakthrough that could lead to further progress on the TPC itself.

With that, we shall end our brief history of this famous conjecture. It has given us the opportunity to meet Alphonse de Polignac and J.W. L. Glaisher, along with Yitang Zhang and the Pentium bug. Compared to Fermat's Last Theorem and the Goldbach conjecture, it is a relatively recent addition to the canon of great problems. But, like both of these, the twin prime conjecture conceals its extraordinary challenges behind a deceptively innocent façade. 